\title{
Room Temperature Scanning Micro-Hall Probe Microscope Imaging of Ferromagnetic Microstructures in the Presence of 2.5 Tesla Pulsed Magnetic Fields Generated by an Integrated Mini Coil
}

\author{
Adarsh SANDHU*, Hiroshi MASUDA ${ }^{1}$ and Ahmet ORAL ${ }^{2}$ \\ Research Centre for Quantum Effect Electronics, Tokyo Institute of Technology, 2-12-1 O-okayama, Meguro-ku, Tokyo 152-8552, Japan \\ ${ }^{1}$ Toei Kogyo Ltd., 8-13-1 Tadao, Machida 194-0035, Japan \\ ${ }^{2}$ Deprtment of Physics, Bilkent University, 06533 Ankara, Turkey
}

(Received September 13, 2002; accepted for publication October 29, 2002)

A unique magnetic imaging system comprising of a room temperature scanning Hall probe microscope with an integrated mini-coil capable of generating pulsed magnetic fields up to 2.5 Tesla (width of 3 ms) was developed for the direct and non-invasive magnetic imaging of ferromagnetic micro-domains in the presence of extremely large external pulsed magnetic fields without adverse vibrational disturbance of the sample during measurements. The system was successfully used for magnetic imaging of the erasure process of bit patterns on the surface of $1.4 \mathrm{MB}$ written floppy disks and the dynamics of micro-domain structures of demagnetized strontium ferrite permanent magnets under large external pulsed magnetic fields. [DOI: 10.1143/JJAP.41.L1402]

KEYWORDS: scanning probe microscopy, pulsed magnetic fields, magnetic imaging, Hall sensors, ferromagnetic micro-domains

A fundamental understanding of the behaviour of magnetic domains in external magnetic fields is important for the development of information technology such as ultra-high density magnetic recording media, high coercivity permanent magnets and multilayered ferromagnetic structures used for fabricating novel 'spintronic' devices. ${ }^{1)}$ We have previously reported on the use of room temperature scanning Hall probe microscopy (RT-SHPM) for the non-invasive and quantitative imaging of localized stray fields at the surfaces of ferromagnetic magnetic microstructures in the presence of static external magnetic fields up to $3000 \mathrm{Oe}^{2-5)}$ However, external fields of more than $2 \mathrm{~T}$ are required to investigate properties such as remanance in high coercivity ferromagnetic structures but it is difficult to achieve fields much larger than $\sim 3000$ Oe using permanent magnets and conventional electromagnets.

The use of pulsed magnetic fields offers a promising method for producing extremely large fields but the integration of pulse coils and peripheral instrumentation with the RTSHPM necessitates extreme care due to the adverse affects of external vibrations during continuous magnetic imaging measurements.

We have succeeded in overcoming these limitations and constructed a new RT-SHPM system incorporating a unique mini-coil capable of generating pulsed magnetic fields up to $2.5 \mathrm{~T}$ (width of $3 \mathrm{~ms}$ ) without vibrational disturbances during imaging when the Hall probe is less than $0.5 \mu \mathrm{m}$ above the sample surface. In this paper we describe the pertinent features of the mini-coil/RT-SHPM system and present experimental results of RT-SHPM measurements on hard disks and strontium ferrite permanent magnetics under external pulsed bias fields demonstrating the feasibility of wider ranging applications of this technology.

Figure 1 is illustrates the main components of the RTSHPM with an integrated mini-coil. RT-SHPM magnetic imaging is carried out using one out of three possible Hall probes (HP) of different sizes and field sensitivities: (i) GaAs/AlGaAs 2-DEG heterostructure, active area of $0.8 \mu \mathrm{m}^{2}$ and sensitivity of $0.04 \mathrm{G} / \sqrt{\mathrm{Hz}}$; (ii) epitaxial $\mathrm{InSb}$, active area

*E-mail address: sandhu@pe.titech.ac.jp

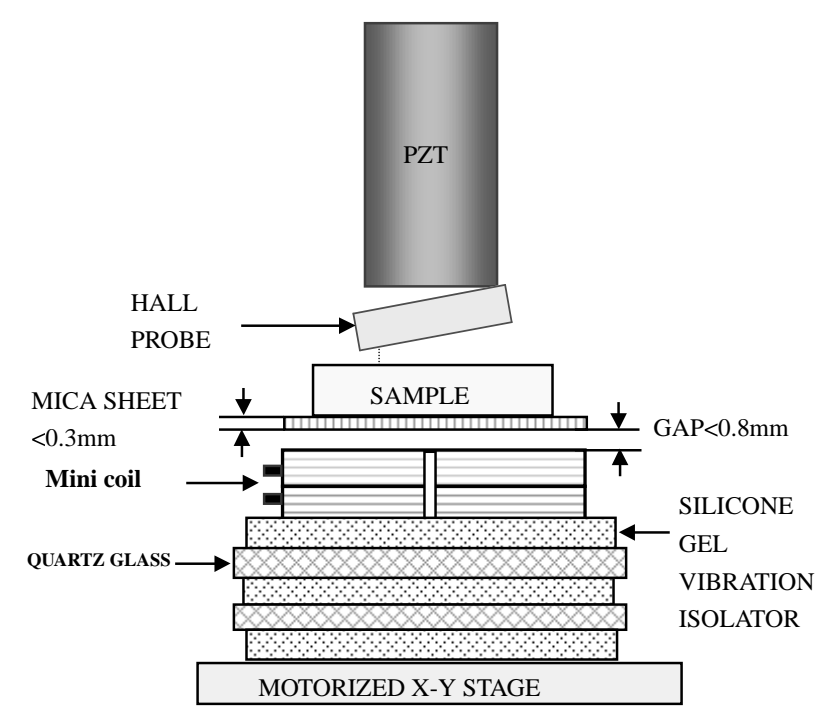

(a)

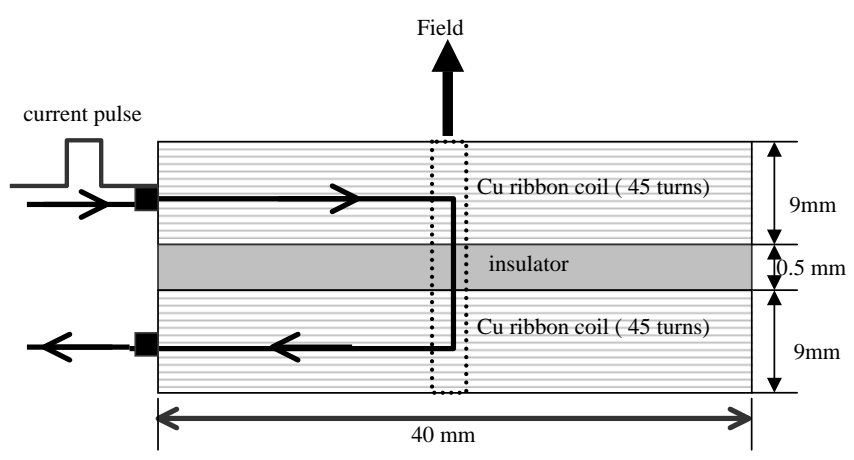

(b)

Fig. 1. (a): Main components of room temperature scanning Hall probe microscope with integrated mini-coil for generating pulsed magnetic fields. (b): Pulsed mini-coil unit consisting of two 'Cu-ribbon' coils connected in series.

of $2 \mu \mathrm{m}^{2}$ and sensitivity of $10 \mathrm{mG} / \sqrt{\mathrm{Hz}}$, (iii) Bi thin film, active area of $0.12 \mu \mathrm{m}^{2}$ and sensitivity of $0.7 \mathrm{G} \sqrt{\mathrm{Hz}}$. The HP is mounted onto a piezoelectric scanning tube (PZT) at a tilt 
angle of $1.5^{\circ}$ with respect to the sample surface. A scanning tunnelling microscope (STM) tip is integrated adjacent to the HP for precise vertical positioning above the sample surface. Magnetic imaging is carried out by scanning the HP over the surface of the sample while simultaneously measuring changes in Hall voltage that are proportional to fluctuations of the perpendicular component of the stray magnetic field emanating from the surface. All measurements in this study were made at a height of $0.5 \mu \mathrm{m}$ above the sample surface. The RT-SHPM enables measurements up to $50 \times 50 \mu \mathrm{m}^{2)}$ and $128 \times 128$ pixel scan data acquisition is possible in about one second.

Figure 1(b) shows an expanded view of the mini-coil integrated with the RT-SHPM system. The pulsed magnetic fields were generated by two spiral shaped ' $\mathrm{Cu}$ ribbon coils' each of 45 turns, wound around a common concentric core and connected in series. The $\mathrm{Cu}$ ribbon used in each coil had a width of $9 \mathrm{~mm}$, a thickness of $200 \mu \mathrm{m}$ and a total length of $5 \mu \mathrm{m}$. The $\mathrm{Cu}$ ribbons making up the coil were electrically insulated using $50 \mu \mathrm{m}$ thick Mylar insulating film and the touching surfaces of the coils are electrically insulated by a $0.5 \mathrm{~mm}$ thick insulator. The use of $\mathrm{Cu}$ ribbon instead of conventional wire coils for fabricating the mini-coil unit has the advantages of a lower electrical inductance, operation at lower voltages and larger current carrying capacity in addition to an easier fabrication procedure. The coil was excited by a pulsed current from a bank of electrolytic capacitors forming part of an electrical circuit that was designed to produce both polarities of perpendicular pulsed magnetic fields with respect to the sample surface.

The data shown in Figs. 2, 3 and 4 were measured using a commercially available, calibrated $0.5 \mathrm{~mm}$ sized GaAs Hall probe. Figure 2 shows the distribution of magnetic field generated by the mini-coil with position as measured by passing a dc current of $30 \mathrm{~A}$ and sensing the resulting field using a calibrated Hall sensor located $1.5 \mathrm{~mm}$ above the coil surface. The magnetic fields shown are average values measured at horizontal intervals of $0.5 \mathrm{~mm}$ over the $40 \mathrm{~mm}$ coil surface and allowance was not made for the variation of the magnetic field over the surface of the Hall probe.

Figure 3 shows the time dependence of the pulsed cur-

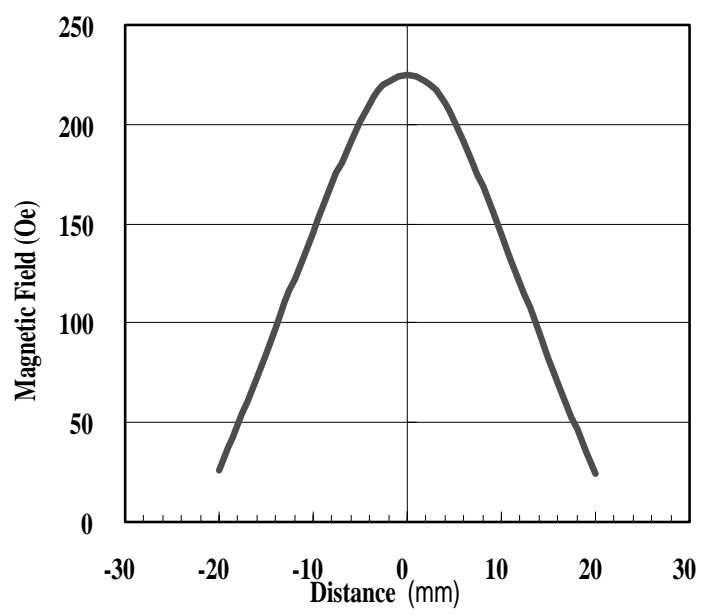

Fig. 2. Distribution of magnetic fields generated by the mini-coil measured by passing a dc current of $30 \mathrm{~A}$ through the coil and using a calibrated Hall sensor positioned $1.5 \mathrm{~mm}$ above the coil surface.

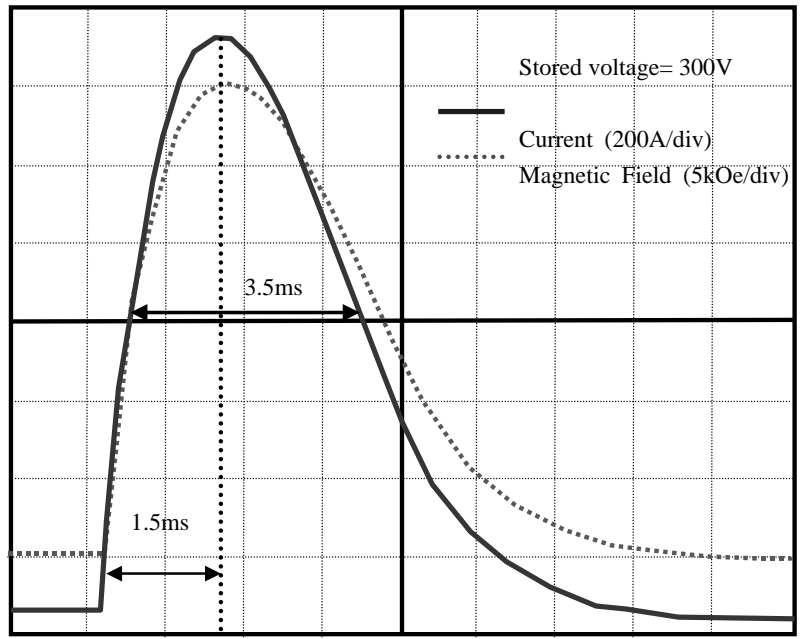

Fig. 3. Time dependence of the pulsed current and corresponding field generated as determined from the output voltage of the Hall sensor.

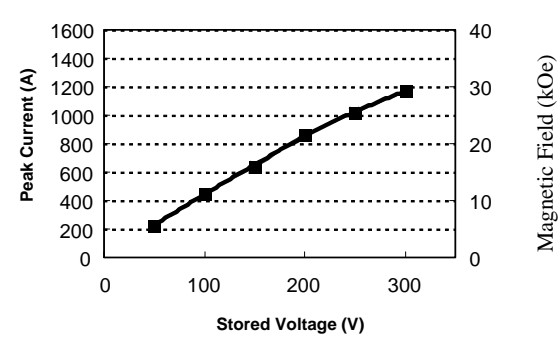

Fig. 4. Variation of peak pulsed current and resulting peak pulsed magnetic field with the stored voltage.

rent and corresponding magnetic field calculated from the output voltage of the Hall sensor. The mini-coil generates pulsed fields with full width at half maximum of approximately $3.5 \mathrm{~ms}$ and a rise time of $1.5 \mathrm{~ms}$.

Figure 4 shows the variation of peak current and magnetic field with the stored voltage. The results show that is it is possible to generate a wide range of pulsed magnetic fields by varying the stored voltage with a maximum of $292 \mathrm{kOe}$ at 300 volts.

The sudden passage of a pulse current through the minicoil induces physical oscillations of the unit as a whole and such vibrations must be de-coupled from the RT-SHPM system to enable continuous, real time measurements under external fields when the separation between the sample surface and Hall probe is $0.5 \mu \mathrm{m}$. The vibrational isolation of the mini-coil unit and sample stage was achieved by resting the mini-coil onto a structure consisting of alternate layers of a soft silicone gel tape ${ }^{6)}$ and quartz glass slab, as shown in Fig. 1(a). The amplitude of vibrations in a direction perpendicular to the sample surface were experimentally verified using a laser Doppler system and were found to range between 0.08 to $0.2 \mu \mathrm{m}$ for excitation pulsed currents between 200-1100 amperes. Such amplitudes of oscillations were sufficiently small to permit continuous measurements even when external pulsed fields were applied with the Hall probe at a distance of $0.5 \mu \mathrm{m}$ above the sample. The perpendicular vibrations had a decay time of $40 \mathrm{~ms}$ and did not adversely affect the resolution of the RT-SHPM since only the initial 30 data points of a $256 \times 256$ sized pixel scan were recorded during the $40 \mathrm{~ms}$ 


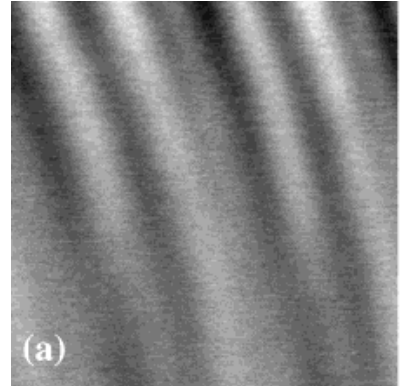

$\mathbf{0} \mathbf{T}$

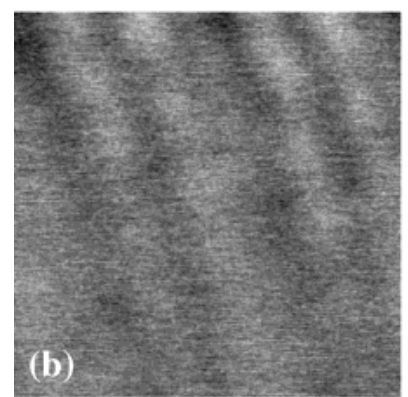

$0.3 \mathrm{~T}$

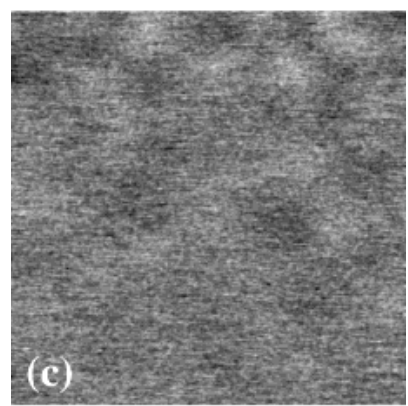

$0.5 \mathrm{~T}$

Fig. 5. (a)-(c) Typical $25 \mu \mathrm{m} \times 25 \mu \mathrm{m}$ RT-SHPM images of a $1.4 \mathrm{MB}$ written floppy disk under external pulsed magnetic fields.

that the sample surface was vibrating perpendicularly.

The mini-coil/RT-SHPM system was used for imaging stray fields at the surfaces of magnetic recording media and permanent magnets. A $0.8 \mu \mathrm{m}^{2} \mathrm{GaAs} / \mathrm{AlGaAs}$ Hall probe was used for all the measurements described hereafter. Figs. 5(a) and 5(c) are typical $25 \mu \mathrm{m} \times 25 \mu \mathrm{m}$ RT-SHPM images of a 1.4 MB written floppy disk (FD) placed in perpendicular external pulsed magnetic fields, where the FD surface was imaged immediately after applying pulsed fields. The black and white regions in the RT-SHPM images represent magnetizations into and out of the plane of the paper. The initial distinct magnetic transitions are seen to deform and coalesce into island-like regions at fields approaching the saturation field of 5000 Oe as verified by VSM measurements. The stray surface fields measured by the RT-SHPM decreased from $\pm 60 \mathrm{G}$ to less than $\pm 10 \mathrm{G}$ as the external pulsed field were increased from 0 to $0.5 \mathrm{~T}$.

Figures 6(a)-6(d) show typical $25 \mu \mathrm{m} \times 25 \mu \mathrm{m}$ images (easy axis normal to the surface) of the variation of stray surface fields with increasing pulsed magnetic fields ap-

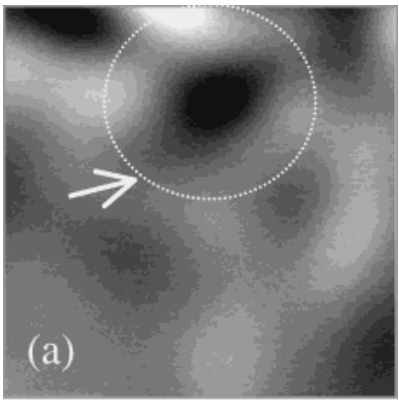

$0 \mathrm{~T}$

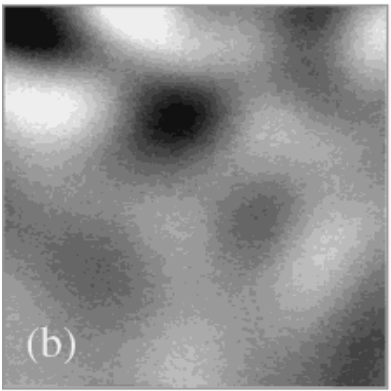

$0.2 \mathrm{~T}$

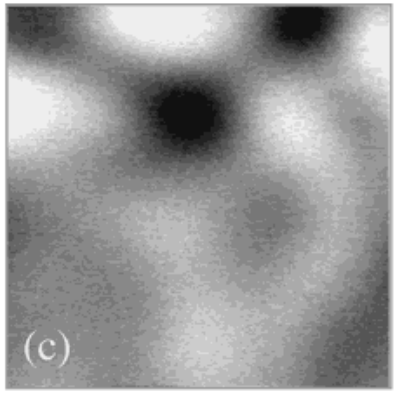

$0.5 \mathrm{~T}$

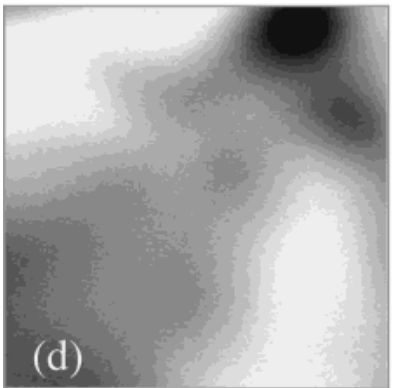

$1.0 \mathrm{~T}$

Fig. 6. (a)-(d) show typical $25 \mu \mathrm{m} \times 25 \mu \mathrm{m}$ images of stray surface fields with increasing pulsed magnetic fields applied parallel to the easy axis of a $200 \mu \mathrm{m}$ thick, demagnetized strontium ferrite permanent magnet sample measuring $5 \mathrm{~mm} \times 5 \mathrm{~mm}$.

plied parallel to the easy axis of a $200 \mu \mathrm{m}$ thick, demagnetized strontium ferrite (SF) permanent magnet sample measuring $5 \mathrm{~mm} \times 5 \mathrm{~mm}$. The sample was prepared by a process involving sintering and had grain sizes ranging between 2-10 micrometers. ${ }^{7-9)}$ The RT-SHPM images show regions on the sample with magnetization perpendicular to its surface due to the existence of clusters of multi-domain grains. Application of external pulsed fields produces domain movement 
followed by rotation and reversal of certain domains at the highest fields as exemplified by region in Fig. 6(a) marked by the broken circle. The gray scale of the images varies between $\pm 200 \mathrm{G}$, from black to white. A study on the physical interpretation of these observations is in progress and the results will be reported elsewhere.

We described the design and construction of a mini-coil unit capable of producing pulsed magnetic fields up to $2.5 \mathrm{~T}$ for integration with a room temperature scanning Hall probe microscopy system. The combined mini-coil/RT-SHPM system was demonstrated to be a valuable instrument for the direct, quantitative and non-invasive observation of localized stray magnetic fields at the surfaces of magnetic recording media and ferromagnetic materials in the presence large external bias fields.

A. S. gratefully acknowledges the support of the
Japanese Ministry of Education, Culture, Sports, Science and Technology (Grant in Aid \#13650354).

1) A. Hubert and R. Schafer: Magnet Domains (Springer, Heidelberg,1998).

2) A. Sandhu, H. Masuda, A. Oral and S. J. Bending: Proc. 8th Asia Pacific Physics Conf., 2000, p. 627.

3) A. Sandhu, H. Masuda, A. Oral and S. J. Bending: Proc. 8th Int. Conf. Ferrite, 2000, p. 390.

4) A. Sandhu, H. Masuda, A. Oral and S. J. Bending: Jpn. J. Appl. Phys. 40 (2001) 4321

5) A. Sandhu, N. Iida, H. Masuda, A. Oral and S. J. Bending: J. Magn. \& Magn. Mater. 242-245 (2002) 1249.

6) 'Aplha-Gel' thin tapes manufactured by Geltech Co. Ltd., Tokyo.

7) H. Taguchi, F. Hirata, T. Takeishi and T. Mori: Proc. Int. Conf. Ferrites (1996) p. 1118.

8) D. Livingston: J. Appl. Phys. 57 (1985) 4137.

9) A. Sandhu, N. Iida, H. Masuda, A. Oral and S. J. Bending: Mater. Res. Soc. Symp. Proc. 674 (2001) U3.2.1. 